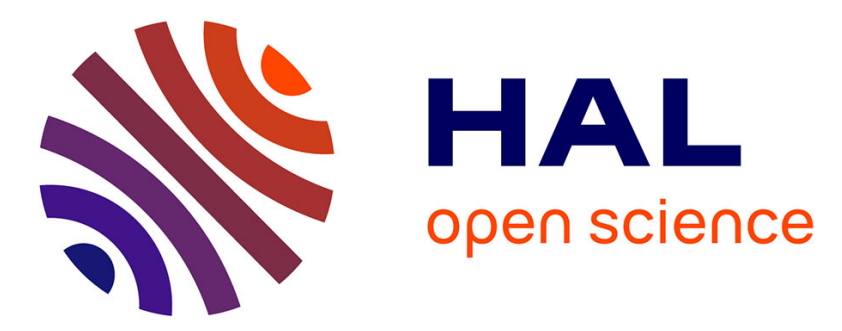

\title{
Appareillage permettant la détermination dans l'ultraviolet lointain des constantes optiques de couches évaporées sous ultra-vide
}

André Daudé, Stéphane Robin

\section{- To cite this version:}

André Daudé, Stéphane Robin. Appareillage permettant la détermination dans l'ultraviolet lointain des constantes optiques de couches évaporées sous ultra-vide. Revue de Physique Appliquée, 1966, 1 (2), pp.120-122. 10.1051/rphysap:0196600102012000 . jpa-00242695

\section{HAL Id: jpa-00242695 https://hal.science/jpa-00242695}

Submitted on 1 Jan 1966

HAL is a multi-disciplinary open access archive for the deposit and dissemination of scientific research documents, whether they are published or not. The documents may come from teaching and research institutions in France or abroad, or from public or private research centers.
L'archive ouverte pluridisciplinaire HAL, est destinée au dépôt et à la diffusion de documents scientifiques de niveau recherche, publiés ou non, émanant des établissements d'enseignement et de recherche français ou étrangers, des laboratoires publics ou privés. 


\title{
APPAREILLAGE PERMETTANT LA DÉTERMINATION DANS L'ULTRAVIOLET LOINTAIN DES CONSTANTES OPTIQUES DE COUCHES ÉVAPORÉES SOUS ULTRA-VIDE
}

\author{
Par André DAUdÉ et Stéphane ROBIN, \\ Laboratoire de Spectroscopie, Faculté des Sciences de Rennes.
}

Résumé. - Nous décrivons un appareil permettant d'évaporer sous ultra-vide $\left(5 \times 10^{-10}\right.$ Torr $)$ un matériau sous forme de couche mince et de faire des mesures optiques dans l'ultraviolet jusqu'à $1200 \AA ̊$. Les couches minces sont étudiées dans l'enceinte où elles ont été réalisées. Nous pouvons mesurer la transmission du rayonnement incident ou sa réflexion suivant deux angles d'incidence ( 20 degrés et 60 degrés) et en déduire les constantes optiques ( $n$, indice de réfraction ; $k$, indice d'extinction) du matériau étudié [1].

\begin{abstract}
A description is given of an apparatus for evaporation under ultra-high vacuum $\left(5 \times 10^{-0}\right.$ Torr $)$ of thin films sample and optical measurements in vacuum ultraviolet to $1200 \AA$ are descrited. The thin films are studied in the chamber where they have been grown. We can measure the transmission or the reflectance of the incident light for two angles of incidence ( 20 degrees and 60 degrees) and deduce the optical constants $(n$, index of refraction; $k$, extinction coefficient) of the sample studied.
\end{abstract}

Description de l'appareillage (fig. 1). - Peu d'appareils permettent actuellement d'effectuer des mesures de pouvoir réflecteur dans l'ultraviolet lointain pour des couches évaporées sous ultra-vide ; à notre connaissance, le seul réflectomètre de ce type [2] a été réalisé par Weissler, mais la transparence des disques de corindon utilisés comme fenêtres limite son emploi à $1500 \AA ̊$.

La figure 1 représente l'enceinte de mesure. Un groupe de pompage à ultra-vide comprenant une pompe ionique à titane de 80 litres/seconde (type Super Vacion de chez Varian) et un évaporateur à titane de 3500 litres/seconde sont raccordés en (A) sur l'ensemble de mesure. Tout l'appareillage est étuvable à $350^{\circ} \mathrm{C}$. Une vanne à ultra-vide (vanne équerre à joint d'indium de chez V. M. D. I.) permet d'isoler l'ensemble d'un groupe de pompage auxiliaire utilisé pendant les étuvages.

Les accessoires nécessaires pour les évaporations et les mesures optiques sont raccordés par joints en cuivre OFHC sur des brides soudées (sous argon; sur le corps principal (B) de l'enceinte. L'ensemble est en acier inoxydable $18 / 8$.

Une jauge Bayard-Alpert (1) indique la pression entre $10^{-3}$ Torr et $10^{-10}$ Torr. Un porte-échantillons (2) peut être déplacé verticalement et orienté suivant différents angles à l'aide d'un aimant extérieur (3), cet aimant doit être retiré pendant les étuvages. Quatre disques (4) en fluorure de lithium permettent la transmission du rayonnement ultraviolet jusqu'à $1140 \AA$ Å environ. L'étanchéité entre ces disques et les embases métalliques qui les supportent est assurée par joints métalliques spéciaux mis au point au laboratoire $\left({ }^{1}\right)$. L'évaporateur (5) est

(1) Une description détaillée de ces joints sera publiée prochainement.

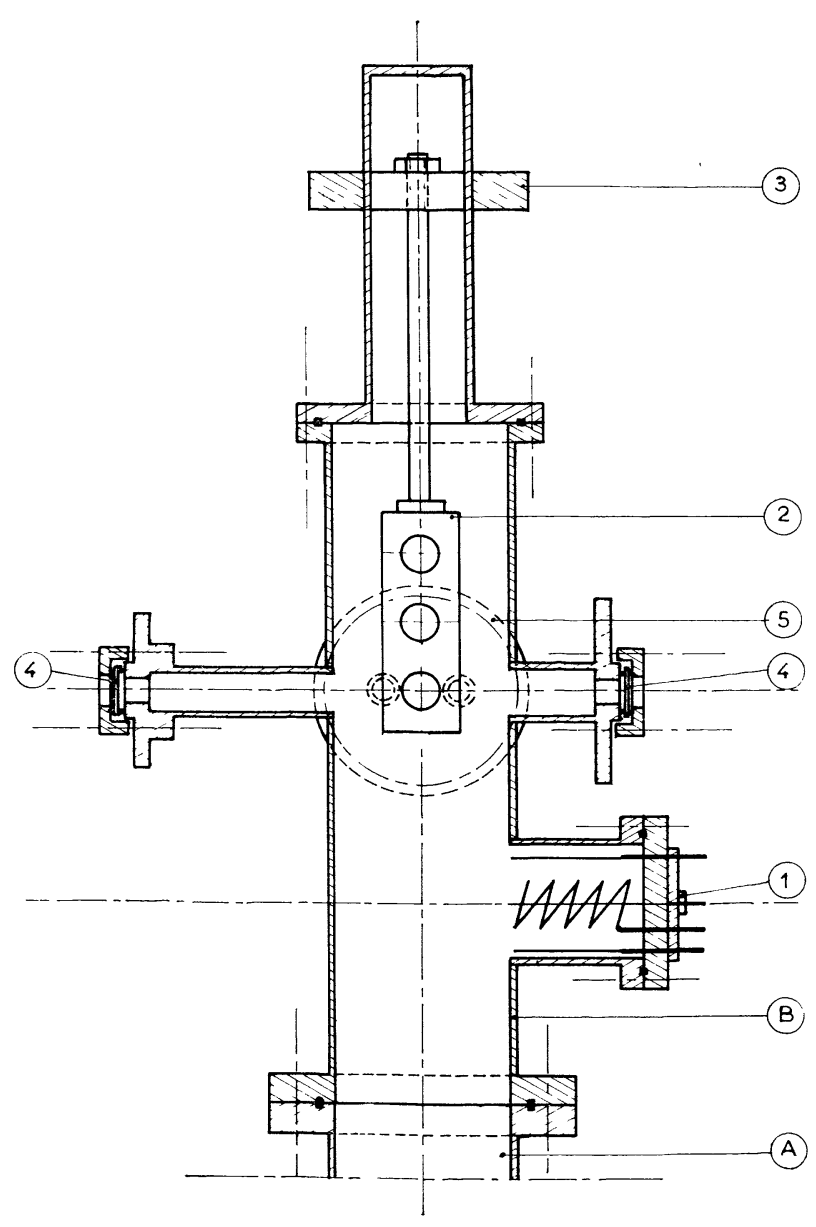

FIG. 1. 
constitué par deux électrodes de cuivre pouvant supporter une intensité maximum de 150 ampères.

Détection. Mesures (fig. 2). - Le réflectomètre est relié en $(\mathrm{F})$ à un monochromateur à réseau en incidence normale [3]. Une lampe à hydrogène sans fenêtre donne un spectre continu de 2400 à $1700 \AA$ et un spectre de raies de 1700 à $1025 \AA$. Le faisceau de lumière $(L)$ issu du monochromateur est divisé en deux parties par un miroir d'aluminium (M).

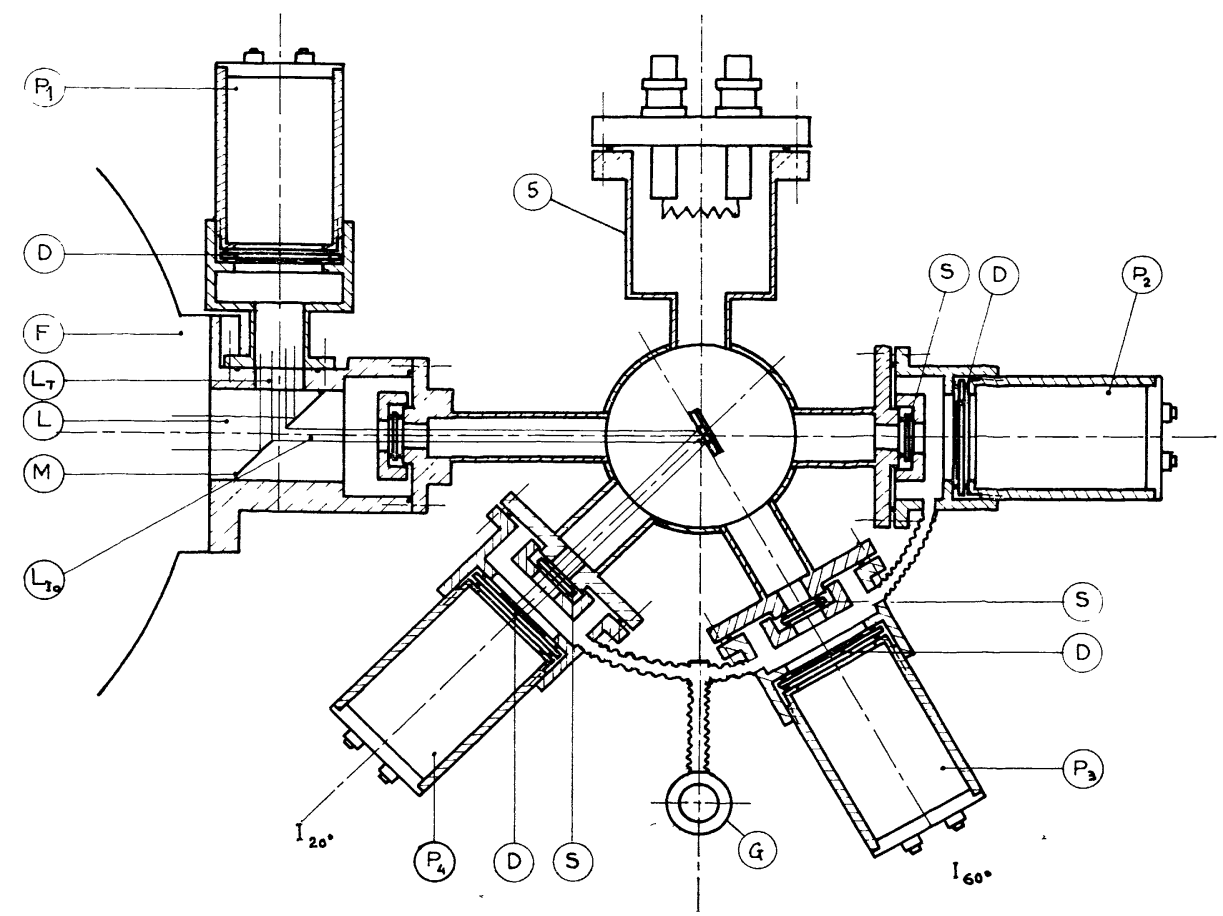

FIG. 2.

Une partie $\left(L_{T}\right)$ est réfléchie par $(M)$ vers un photomultiplicateur témoin $\left(\mathrm{P}_{1}\right)$ et sert à contrôler la stabilité de la source durant les mesures. La seconde partie constitue le faisceau $\left(\mathrm{L}_{\mathrm{T}_{o}}\right)$ utilisé dans le réflectomètre. On mesure le rayonnement témoin $\left(\mathrm{L}_{\mathrm{T}}\right)$, direct $\left(\mathrm{L}_{\mathrm{I}_{0}}\right)$ ou réfléchi sous des incidences de 20 degrés $\left(\mathrm{I}_{20^{\circ}}\right)$ ou 60 degrés $\left(\mathrm{I}_{60} \mathrm{o}\right)$ à l'aide de photomultiplicateurs Radiotechnique 150 A V P placés derrière un disque de pyrex (D) recouvert d'une mince couche de terphényl [4]. Un groupe de pompage raccordé en $G$ maintient un vide inférieur à 10-4 torr entre les fenêtres de sortie du réflectomètre (S) et la substance fluorescente (D) afin de permettre la transmission des rayonnements de longueur d'onde inıérieure à 2000. .

Pour déterminer les valeurs du pouvoir réflecteur des couches minces réalisées dans notre appareil nous utilisons une lame de référence dont nous avons préalablement mesuré avec soin le pouvoir réflecteur dans le domaine spectral étudié. A cet effet, nous avons utilisé des disques de quartz, de corindon taillés perpendiculairement à l'axe optique, une couche épaisse stabilisée d'or.

Résultats obtenus. - Pour tester notre appareil, nous avons choisi l'aluminium qui a déjà fait l'objet de nombreuses études dans l'ultraviolet [5] et permet plusieurs points de comparaison. Une étude très détaillée de ce corps a été effectuée par Madden [6] dans les conditions suivantes : pression limite avant évaporation $2 \times 10^{-7}$ torr, pression durant l'évaporation $5 \times 10^{-6}$ torr, vitesse de dépôt $1000 \AA / \mathrm{s}$. Notre ensemble nous a permis d'obtenir, une pression de $5 \times 10^{-10}$ torr avant évaporation, une pression comprise entre 5 et $8 \times 10^{-8}$ torr pendant la formation de la couche, un retour à une pression inférieure à $5 \times 10^{-9}$ torr 15 secondes après l'évaporation, des vitesses de dépôt de $40 \AA /$ sec à $100 \AA / \mathrm{s}$. L'aluminium utilisé était pur à $99,999 \%$.

Les réglages optiques sont faits avant l'évaporation. Nous avons pu commencer les mesures une minute après la formation de la couche. La courbe 1, (fig. 3) résume les résultats obtenus avec des couches d'aluminium de 600 à $900 \AA$ d'épaisseur étudiées sous une incidence de 20 degrés. La précision des mesures est évaluée à $\pm 2,5 \%$. Les mesures ont été effectuées avec la raie Lyman $\alpha$ de l'hydrogène $1216 \AA$. La courbe 2 , fig. 3 , représente les résultats antérieurs de Madden dans les conditions énumérées ci-dessus. Nous notons une amélioration de la valeur du pouvoir réflecteur, une décroissance 


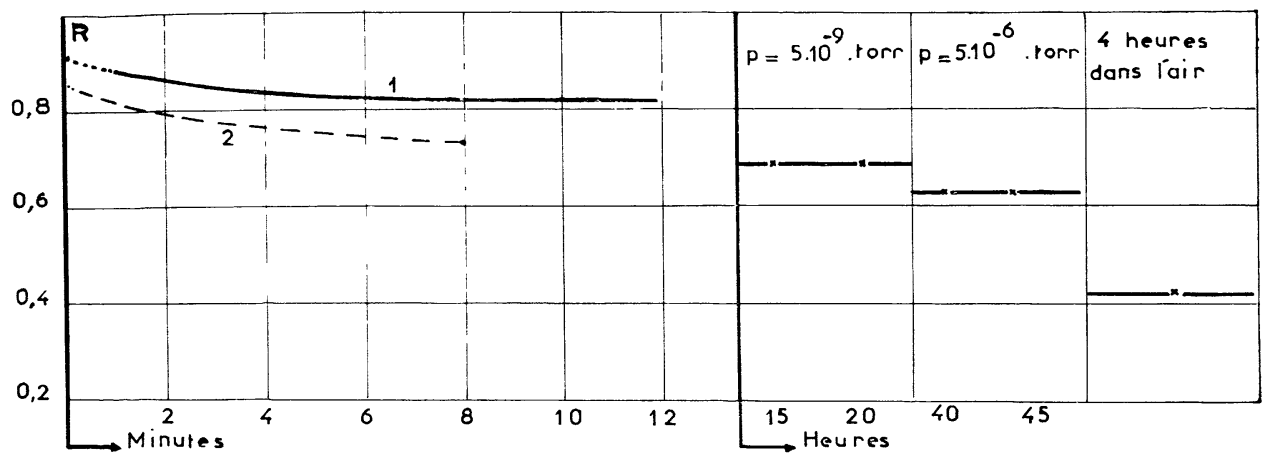

FIG. 3.

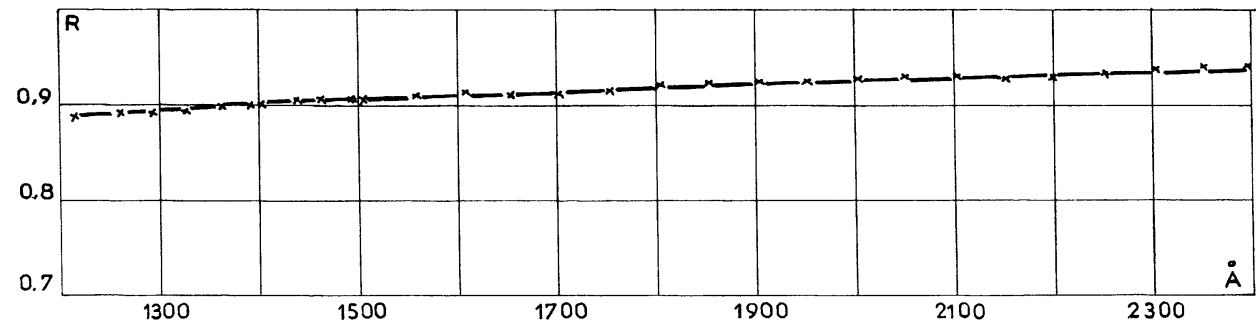

FIG. 4.

nettement plus faible en fonction du temps. La valeur extrapolée du pouvoir réflecteur au temps zéro est évaluée à $90 \%$ environ.

La figure 4 résume les valeurs du pouvoir réflecteur obtenues pour différentes longueur d'onde. L'enregistrement des mesures a commencé une minute après la fin de l'évaporation et a duré $15 \mathrm{mi}$ nutes pour l'exploration du domaine spectral compris entre 1216 et $2400 \AA$. Compte tenu de la durée de l'enregistrement, les valeurs indiquées doivent être légèrement inférieures à celles qui auraient été obtenues une minute seulement après formation de la couche.

Ces premières mesures ont permis de mettre en évidence une amélioration sensible du pouvoir réflecteur de l'aluminium évaporé sous ultra-vide dans l'ultraviolet lointain. Le calcul des valeurs correspondantes de $n$ et $k$ fera l'objet d'une publication ultér.eure.

Manuscrit reçu le 8 mars 1966.

\section{BIBLIOGRAPHIE}

[1] Tousey (R.), J. O. S. A., 1939, 29, 235.

[2] Weissler (G. L.), Technical Report No - USC. VAC. UV 104, août 1965.

[3] Romand (J.) et Vodar (B.), Res. Opt., 1960, 39, 143.

[4] Lemonnier (J. C.), Priol (M.), Quemerais (A.) et
Roвin (Mme S.), J. Physique, 1964, 25, 79 A.

[5] Madden (R. P.), Canfield (L. R.) et Hass (G.), J. O. S. A., 1963, 53, 620 .

[6] Hass (G.), Physics of thin films, Academic Press, 1963, p. 123. 